\title{
Characterization of Power Function Distribution through Expectation of Function of Order Statistics
}

\author{
Bhatt Milind B.
}

Department of Statistics, Sardar Patel University, Vallabh Vidyanagar-388120, Dist: Anand, State: Gujarat, India

Copyright (C) 2015 by authors, all rights reserved. Authors agree that this article remains permanently

open access under the terms of the Creative Commons Attribution License 4.0 International License

\begin{abstract}
Independence of suitable function of order statistics, linear relation of conditional expectation, recurrence relations between expectations of function of order statistics, distributional properties of exponential distribution, record valves, lower record statistics, product of order statistics and Lorenz curve etc.. are various approaches available in the literature for the characterization of the power function distribution. In this research note different path breaking approach for the characterization of power function distribution through the expectation of function of order statistics is given and provides a method to characterize the power function distribution which needs any arbitrary non constant function only.
\end{abstract}

Keywords Characterization, Power function distribution, Probability Density Function.

\section{Introduction}

Notable attempt to characterized Power function distribution through independence of suitable function of order statistics and distributional properties of transformation of exponential are Basu [1], Govindarajulu [2], Desu [3] and Dallas [4] where as of exponential and related distributions assuming linear relation of conditional expectation by Beg [5], characterization based on record values by Nagraja [6], characterization of some types of distributions using recurrence relations between expectations of function of order statistics by Alli [7], characterization results on exponential and related distributions by Tavangar [8] and characterization of continuous distributions through lower record statistics by Faizan [9] included the characterization of power function distribution as special case.

Direct characterization for power function distribution has been given in Fisz [10] who use independence properties of order statistics where as Arslan [11] used product of order statistic. [contraction is a particular case of product of order statistics which has interesting pplications such as in economic modeling and reliability see Alamatsaz [12], Kotz [13] and Alzaid [14] ] where as Moothathu [15] used Lorenz curve. [Graph of fraction of total income owned by lowest pth fraction of the population is Lorenz curve of distribution of income of distribution of income.][ See. Kendall and Stuart [16]].

This research note provides the characterization based on identity of distribution and equality of expectation of function of order statistics for power-function distribution with the probability density function (p.d.f.)

$f(x ; \theta)= \begin{cases}c \theta^{-c} x^{c-1} ; & a<x<\theta<b ; \theta=k^{-1}, k>0, c>0, \\ 0, & \text { otherwise }\end{cases}$

where $-\infty<a<b<\infty$ are known constants, $x^{c-1}$ is positive absolutely continuous function and $\left(\frac{c}{\theta}\right)^{c}$ is everywhere differentiable function. Since derivative of $x^{c-1}$ being positive and since range is truncated by $\theta$ from right for $f(x ; \theta)$ defined in (1.1), $\frac{a^{c}}{c}=0$.

The aim of the present research note is to give the new characterization through the expectation of function of order statistics, using identity and equality of expectation. Characterization theorem derived in section 2 with method for characterization as remark and section 3 devoted to applications for illustrative purpose.

\section{Characterization theorem}

\section{Theorem}

Let $X_{1}, X_{2}, X_{n}$ be a random sample of size $n$ from distribution function $F$. Let $X_{1: n}<X_{2: n}, \ldots,<X_{n: n}$ be the set of corresponding order statistics. Assume that

$F$ is continuous on the interval $(a, b)$ where $-\infty<a<$ $b<\infty$. Let $g\left(X_{n: n}\right)$ and $\phi\left(X_{n: n}\right)$ be be two distinct 
differentiable and intregrable functions of $n^{t h}$ order statistic; $X_{n: n}$ on the interval $(a, b)$ where $-\infty<a<b<\infty$ and moreover $g\left(X_{n: n}\right)$ be non-constant function of $X_{n: n}$. Then

$$
E\left[g\left(X_{n: n}\right)+\left(\frac{X_{n: n}}{n c}\right) \frac{d}{d X_{n: n}} g\left(X_{n: n}\right)\right]=g(\theta) .
$$

is the necessary and sufficient condition for pdf $f(x ; \theta)$ of $F$ to be $f(x ; \theta)$ defined in (1).

\section{Proof}

Given $f(x ; \theta)$ defined in (1), for necessity of $(2)$ if $\phi\left(X_{n: n}\right)$ is such that $g(\theta)=E\left[\phi\left(X_{n: n}\right)\right]$ where $g(\theta)$ is differentiable function then using $f\left(x_{n: n} ; \theta\right)$; pdf of $n^{t h}$ order statistic one gets,

$$
g(\theta)=\int_{a}^{\theta} \phi\left(x_{n: n}\right) f\left(x_{n: n} ; \theta\right) \mathrm{d} x_{n: n} .
$$

Differentiating (3) with respect to $\theta$ on both sides and replacing $X_{n: n}$ for $\theta$, and simplifying one gets

$$
\phi\left(X_{n: n}\right)=g\left(X_{n: n}\right)+\left(\frac{X_{n: n}}{n c}\right) \frac{d}{d X_{n: n}} g\left(X_{n: n}\right),
$$

which establishes necessity of (2). Conversely given (2), let $k\left(x_{n: n} ; \theta\right)$ be the p.d.f. of pdf of $n^{t h}$ order statistic such that

$$
\begin{aligned}
g(\theta)= & \int_{a}^{\theta}\left[g\left(x_{n: n}\right)+\left(\frac{x_{n: n}}{n c}\right) \frac{d}{d x_{n: n}} g\left(x_{n: n}\right)\right] \\
& . k\left(x_{n: n} ; \theta\right) \mathrm{d} x_{n: n},
\end{aligned}
$$

Since $\left(\frac{c}{x_{n: n}}\right)^{n}$ is decreasing integrable and differentiable function on the interval $(a, b)$ with $\left(\frac{a}{c}\right)^{n}=0$, the following identity holds

$$
g(\theta) \equiv\left(\frac{c}{\theta^{c}}\right)^{n} \int_{a}^{\theta}\left[\frac{d}{d x_{n: n}} g\left(x_{n: n}\right)\left(\frac{x_{n: n}^{c}}{c}\right)^{n}\right] \mathrm{d} x_{n: n} .
$$

Differentiating integrand $g\left(x_{n: n}\right)\left(\frac{x_{n: n}^{c}}{c}\right)^{n}$ with respect to $x_{n: n}$ and simplifying after taking $\frac{d}{d x_{n: n}}\left(\frac{x_{n: n}^{c}}{c}\right)^{n}$ as one factor one gets (6) as

$$
\begin{aligned}
g(\theta) \equiv & \int_{a}^{\theta}\left[g\left(x_{n: n}\right)+\frac{\left(\frac{x_{n: n}^{c}}{c}\right)^{n}}{\frac{d}{d x_{n: n}}\left(\frac{x_{n: n}^{c}}{c}\right)^{n}} \frac{d}{d X_{n: n}} g\left(X_{n: n}\right)\right] \\
& \cdot\left[\left(\frac{c}{\theta^{c}}\right)^{n} \frac{d}{d x_{n: n}}\left(\frac{x_{n: n}^{c}}{c}\right)^{n}\right] \mathrm{d} x_{n: n}
\end{aligned}
$$

Substituting derivative of $\left(\frac{x_{n: n}^{c}}{c}\right)^{n}$ in (7) one gets (7) as

$$
g(\theta) \equiv \int_{a}^{\theta} \phi\left(x_{n: n}\right)\left(\frac{n c}{\theta^{n c}} x_{n: n}^{n c-1}\right) \mathrm{d} x_{n: n}
$$

where $\phi\left(X_{n: n}\right)$ is as derived in (4). By uniqueness theorem from (5) and (8)

$$
k\left(x_{n: n} ; \theta\right)=\left(\frac{n c}{\theta^{n c}} x_{n: n}^{n c-1}\right) .
$$

Since $\left(\frac{c}{x_{n: n}}\right)^{n}$ is decreasing integrable and differentiable function on the interval $(a, b)$ with $\left(\frac{a}{c}\right)^{n}=0$ and since $\left(\frac{c}{x_{n: n}}\right)^{n}$ is decreasing function for $-\infty<a<b<\infty$ and $\left(\frac{a}{c}\right)^{n}=0$ is satisfy only when range of $x_{n: n}$ is truncated by $\theta$ from right and integrating (9) on the interval $(a, \theta)$ on both sides, one gets

$$
1=\int_{a}^{\theta} k\left(x_{n: n} ; \theta\right) \mathrm{d} x_{n: n}
$$

For $n=1$, in $(10),\left[k\left(x_{n: n} ; \theta\right)\right]_{n=1}$ reduces to $f(x ; \theta)$ defined in (1). Hence sufficiency of (2) is established.

\section{Remark 2.1}

Using $\phi\left(X_{n: n}\right)$ derived in $(4)$, the $f(x ; \theta)$ given in (1) can be determined by

$$
M\left(X_{n: n}\right)=\frac{\frac{d}{d X_{n: n}} g\left(X_{n: n}\right)}{\left.\phi\left(X_{n: n}\right)-g\left(X_{n: n}\right)\right)} .
$$

and pdf is given by

$$
f(x ; \theta)=\left[\frac{\frac{d}{d X_{n: n}} T\left(X_{n: n}\right)}{T(\theta)}\right]_{n=1} .
$$

where $T\left(X_{n: n}\right)$ is increasing function for $-\infty<a<b<\infty$ with $T(a)=0$ such that it satisfies

$$
M\left(X_{n: n}\right)=\frac{d}{d X_{n: n}} \log \left[T\left(X_{n: n}\right)\right] .
$$

\section{Remark 2.2}

The theorem 2.1 for function of $n^{\text {th }}$ order statistics with remark 2.1 also holds for random variable $\mathrm{X}$ when $n=1$ (see Bhatt [17]). 


\section{$3 \quad$ Illustrative Examples}

\section{Examples}

Using method describe in remark 2.1 power function distribution through expectation of non-constant function of order statistics is characterized for illustrative example and significant of unified approach of characterization result.

Example 3.1 Characterization of power function distribution through the Minimum Variance Unbiased (UMVU) estimator $\widehat{\theta^{r}}$ of $\theta^{r}$ is given.

$$
\widehat{\theta^{r}}=\left(1+\frac{r}{c n}\right) X_{n: n}^{r}=g\left(X_{n: n}\right)
$$

Using (4) one gets

$$
\phi\left(X_{n: n}\right)=\left(1+\frac{r}{n c}\right)^{2} X_{n: n}^{r}
$$

and (11) of remark 2.1 will be

$$
M\left(X_{n: n}\right)=\frac{\frac{d}{d X_{n: n}} g\left(X_{n: n}\right)}{\left.\phi\left(X_{n: n}\right)-g\left(X_{n: n}\right)\right)}=\frac{n c}{X_{n: n}} .
$$

with

$$
\frac{d}{d X_{n: n}} \log \left(\frac{X_{n: n}^{c}}{c}\right)^{n}=M\left(X_{n: n}\right)
$$

then

$$
T\left(X_{n: n}\right)=\left(\frac{X_{n: n}^{c}}{c}\right)^{n}
$$

and

$$
f(x ; \theta)=\left[\frac{\frac{d}{d x_{n: n}} T\left(x_{n: n}\right)}{T(\theta)}\right]_{n=1}=c \theta^{-c} x^{c-1} .
$$

Example 3.2 Characterization of power function distribution through the uniformly minimum variance unbiased (UMVU) estimator $\widehat{g}(\theta)$ and maximum likelihood estimator (MLE) $\widetilde{g}(\theta)$ of $g(\theta)$ such as mean; $\mu_{1}^{\prime}(\theta)$, rth moment; $\mu_{r}^{\prime}(\theta), e^{\theta}, e^{-\theta}, p^{t h}$ quantile; $Q_{p}(\theta)$, distribution function; $F(t)$; reliability function; $\bar{F}(t)$, hazard rate; $\lambda(t)$ is given. The UMVU estimator

$\widehat{g}(\theta)= \begin{cases}\widehat{\mu_{1}^{\prime}(\theta)}=\left(c+\frac{1}{n}\right) \frac{X_{n: n}}{c+1} ; & \text { for } \mathrm{i}=1, \\ \widehat{\mu_{r}^{\prime}(\theta)}=\left(c+\frac{r}{n}\right) \frac{X_{n: n}^{r}}{c+r} ; & \text { for } \mathrm{i}=2, \\ \widehat{e^{\theta}}=\left[1+\frac{X_{n: n}}{n c}\right] e^{X_{n: n}} ; & \text { for } \mathrm{i}=3, \\ \widehat{e^{-\theta}}=\left[1-\frac{X_{n: n}}{n c}\right] e^{-X_{n: n}} ; & \text { for } \mathrm{i}=4, \\ \widehat{Q_{p}}(\theta)=\left(1+\frac{1}{n c}\right) p^{-\frac{1}{c}} ; & \text { for } \mathrm{i}=5, \\ \widehat{F}(t)=\left(1-\frac{1}{n}\right)\left(\frac{t}{X_{n: n}}\right)^{c} ; & \text { for } \mathrm{i}=6, \\ \widehat{\bar{F}}(t)=1-\left(1-\frac{1}{n}\right)\left(\frac{t}{X_{n: n}}\right)^{c} ; & \text { for } \mathrm{i}=7, \\ \widehat{\lambda}(t)=-\left[n\left(\left(\frac{t}{X_{n: n}}\right)^{c}-1\right)\right]^{-2} & \\ \cdot\left[n-1+n\left(\frac{t}{X_{n: n}}\right)^{c}\right] & \\ \cdot\left(\frac{c}{t}\right)\left(\frac{t}{X_{n: n}}\right)^{c} ; \text { for } \mathrm{i}=7, & \end{cases}$

and MLE

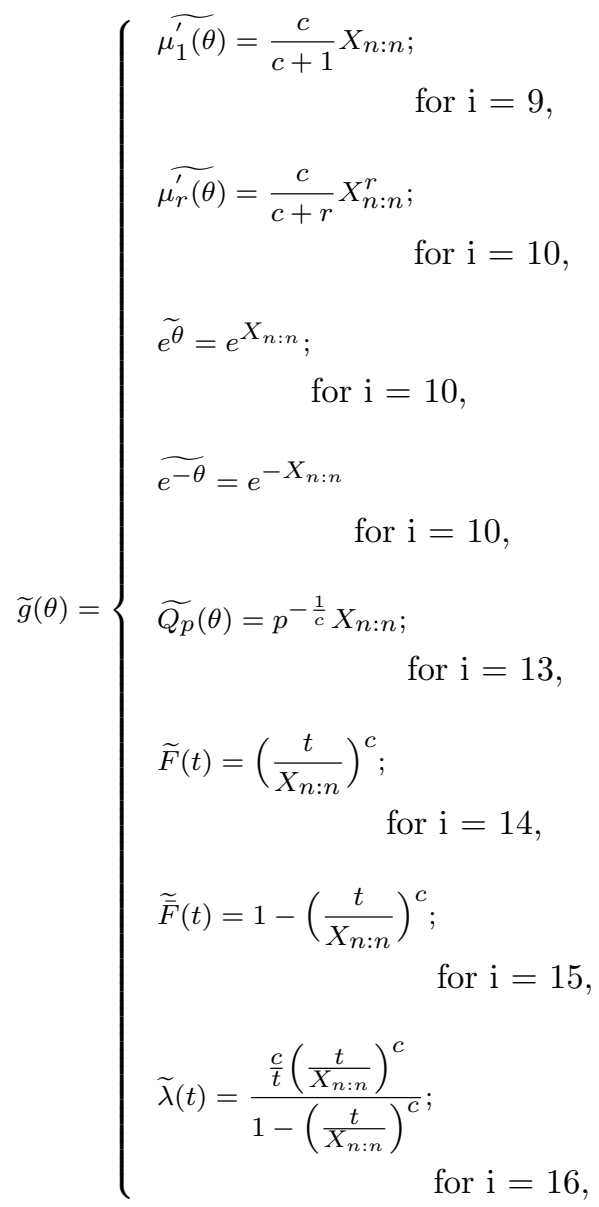




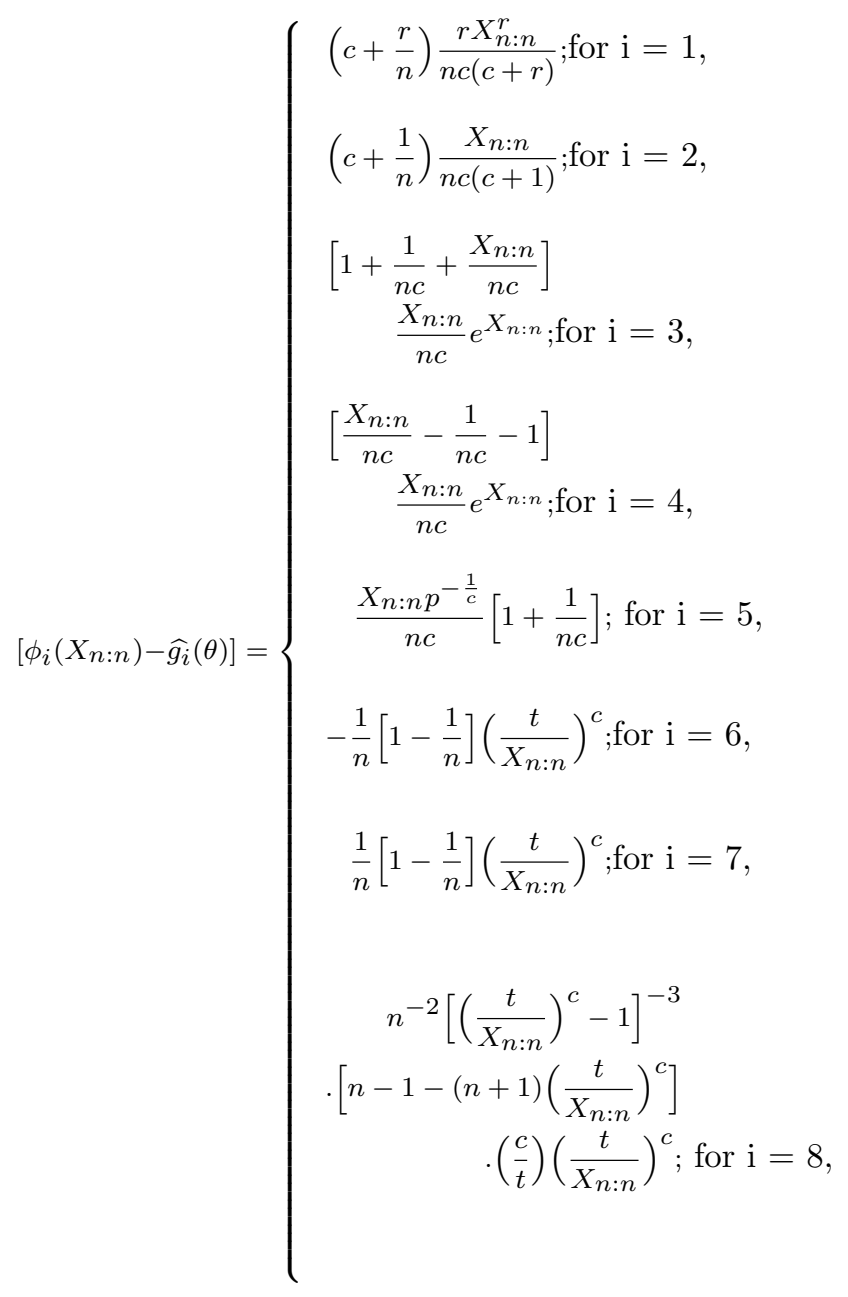

and

$\left[\phi_{i}\left(X_{n: n}\right)-\widetilde{g}_{i}(\theta)\right]=\left\{\begin{array}{c}\frac{X_{n: n}}{n(c+1)} ; \text { for } \mathrm{i}=9, \\ \frac{r X_{n: n}^{r}}{n(c+r)} ; \text { for } \mathrm{i}=10, \\ \frac{X_{n: n}}{n c} e^{-X_{n: n}} ; \text { for } \mathrm{i}=11, \\ -\frac{X_{n: n}}{n c} e^{-X_{n: n}} ; \text { for } \mathrm{i}=12, \frac{X_{n: n} P^{-\frac{1}{c}}}{n c} ; \text { for } \mathrm{i}=13, \\ -\frac{1}{n}\left(\frac{t}{X_{n: n}}\right)^{c} ; \text { for } \mathrm{i}=14, \\ \frac{1}{n}\left(\frac{t}{X_{n: n}}\right)^{c} ; \text { for } \mathrm{i}=15, \\ \frac{c}{t}\left(\frac{t}{X_{n: n}}\right)^{c}\end{array}\right.$
Then by defining $M\left(X_{n: n}\right)$ given in (11) and substituting $T\left(X_{n: n}\right)$ as appeared in (13) for $(12), f(x ; \theta)$ is characterized.

Example 3.3 In context of remark 2.2 characterization of power function distribution through hazard rate; $\lambda(\theta)$ is given.

$$
\lambda(\theta)=\frac{\frac{c}{t}\left(\frac{t}{\theta}\right)^{c}}{1-\left(\frac{t}{X}\right)^{c}}
$$

Therefore

$$
g(X)=\frac{\frac{c}{t}\left(\frac{t}{X}\right)^{c}}{1-\left(\frac{t}{X_{n: n}}\right)^{c}}
$$

and from (4)

$$
\phi(X)=g(X)+\left(\frac{X}{c}\right) \frac{d}{d X} g(X)=\frac{\left(\frac{c}{t}\right)\left(\frac{t}{X}\right)^{2 c}}{\left[\left(\frac{t}{X}\right)-1\right]^{2}}
$$

and (11) of remark 2.1 will be

$M(X)=\frac{\frac{d}{d X} g(X)}{\phi(X)-g(X)}=\frac{c}{x}$

with

$\frac{d}{d X} \log \left[\frac{X^{c}}{c}\right]=M(X)$

then

$T(X)=\frac{X^{c}}{c}$.

and

$f(x ; \theta)=\left[\frac{\frac{d}{d x} T(x)}{T(\theta)}\right]=c \theta^{-c} x^{c-1}$

Example 3.4 Using remark 2.2 the pdf $f(x ; \theta)$ defined in (1) can be characterized through non constant functions of $\theta$ such as

$$
g_{i}(\theta)=\left\{\begin{array}{l}
\frac{c}{c+1} \theta ; \text { meanfor } \mathrm{i}=9, \\
\frac{c}{c+r} \theta_{n: n}^{r} ; r^{t h} \text { rowmomentfor } \mathrm{i}=10, \\
e^{\theta} ; \text { for } \mathrm{i}=10, \\
e^{-\theta} \text { for } \mathrm{i}=10, \\
p^{-\frac{1}{c} \theta} ; p^{t h} \text { quantilefor } \mathrm{i}=13, \\
\left(\frac{t}{\theta}\right)^{c} ; \text { distributionfunctionfor } \mathrm{i}=14, \\
1-\left(\frac{t}{\theta}\right)^{c} ; \text { reliabilityfor } \mathrm{i}=15, \\
\frac{c}{t}\left(\frac{t}{\theta}\right)^{c} \\
1-\left(\frac{t}{X}\right)^{c} ; \text { for } \mathrm{i}=16,
\end{array}\right.
$$

respectively. 
by using

$$
\left[\phi_{i}(X)-g_{i}(X]=\left\{\begin{array}{c}
\frac{X}{c+1} ; \text { for } \mathrm{i}=9, \\
\frac{c X^{r}}{c+r} ; \text { for } \mathrm{i}=10, \\
\frac{X}{c} e^{X} ; \text { for } \mathrm{i}=11, \\
-\frac{X}{c} e^{-X} ; \text { for } \mathrm{i}=12, \\
\frac{X P^{-\frac{1}{c}}}{c} ; \text { for } \mathrm{i}=13, \\
-\left(\frac{t}{X}\right)^{c} ; \text { for } \mathrm{i}=14, \\
\frac{\left(\frac{t}{X}\right)^{c} ; \text { for } \mathrm{i}=15,}{\frac{c}{t}\left(\frac{t}{X}\right)^{c}}\left[\begin{array}{c}
\left.\left.\frac{t}{X}\right)^{c}\right]^{2} \\
{\left[1-\left(\frac{1}{1}\right)\right.}
\end{array},\right.
\end{array}\right.\right.
$$

defining $M(X)$ given in (11) and substituting $T(X)$ as appeared in (13) for $(12), f(x ; \theta)$ is characterized.

\section{Acknowledgements}

This work was supported by UGC Major Research Project : F.No.42-39/2013(SR) dated 12-3-2013.

\section{REFERENCES}

[1] A. P. Basu. On characterizing the exponential distribution by order statistics, Ann. Inst. Statist. Math.,17, 93-96, 1965.

[2] Z. Govindarajulu. Characterization of exponential and power distribution, Skand, Aktuarietidskr., 49, 132-136,1966.
[3] ]M. M. Desu. A characterization of the exponential distribution by order statistics.Ann. Math. Stat., 42, 837 - 838. 1971.

[4] ] A. C. Dallas. Characterization Pareto and power distribution, Ann. Ins. Statist. Math, 28, Part A, pp. 491 - 497, 1976 .

[5] M. L. Beg and Kirmani, S. N. U. A. on a characterization of exponential and related distributions, Austral. J. Statist., 16 (3), 163-166, 1974.

[6] H. N. Nagaraja. On a characterization based on record valves Austral. J. Statist., 19 (1), 70-73, 1977.

[7] M. A. Ali, and A. H. Khan. Characterization of Some Types of Distributions, Information and Management Sciences, Vol. 9, No. 2, June, 1998.

[8] M. Tavangar and M. Asadi. some new characterization results on exponential and related distributions, Bulletin of the Iranian Mathematical Society Vol. 36 No. 1, 257-272, 2010.

[9] M. Faizan and M. I. Khan. A Characterization of Continuous Distributions through Lower Record Statistics, ProbStat Forum, Volume 04, Pages 39-43, April 2011.

[10] M. Fisz. Characterization of some probability distribution,Skand. Aktuarietidskr, 41, 65-67, 1958.

[11] G. Arslan. Characterization based on product of order statistics, math.ST., arXiv 1110.2879v 1, 2011.

[12] M. H. Alamatsaz. Separation of charged and neutral current events from background, proceeding of annual physics conference of Iran, Isfahan University of Technology,1985.

[13] S. Kotz and F. W. Steutel. Note on a characterization of exponential distributions. Stat. Probab. Lett. 6, 201$203,1988$.

[14] A. A. Alzaid and M. A. Al-Osh. An Integer-Valued pthOrder Autoregressive Structure (INAR(p)) Process. J. Appl. Prob., 27: 314-324, 1990.

[15] T. S. K. Moothathu. Characterization of power function distribution through property of Lorenz curve, Sankhya ?, Indian Journal of Statistics, Volume 48, Series B, Pt. 2, 262-265, 1986.

[16] M. G. Kendall and A. Stuart. The Advanced Theory of Statistics,London, Griffin, 1952.

[17] M. B. bhatt. Characterization of Power-Function Distribution through Expectation, Open Journal of Statistics, 3, 441-443, 2013. 